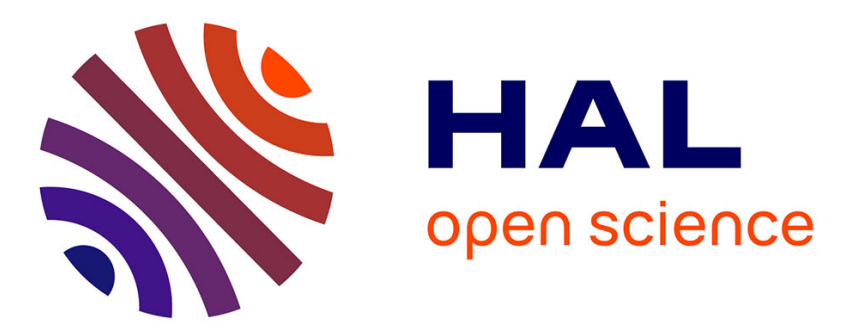

\title{
Effect of structural considerations on the development of free energy functionals for the square-well fluid
}

Thomas Bernet, Manuel Pineiro, Frédéric Plantier, Christelle Miqueu

\section{To cite this version:}

Thomas Bernet, Manuel Pineiro, Frédéric Plantier, Christelle Miqueu. Effect of structural considerations on the development of free energy functionals for the square-well fluid. Molecular Physics, 2018, 116 (15-16), pp.1977-1989. 10.1080/00268976.2018.1438677 . hal-02013246

\section{HAL Id: hal-02013246 \\ https://hal-univ-pau.archives-ouvertes.fr/hal-02013246}

Submitted on 2 Apr 2019

HAL is a multi-disciplinary open access archive for the deposit and dissemination of scientific research documents, whether they are published or not. The documents may come from teaching and research institutions in France or abroad, or from public or private research centers.
L'archive ouverte pluridisciplinaire HAL, est destinée au dépôt et à la diffusion de documents scientifiques de niveau recherche, publiés ou non, émanant des établissements d'enseignement et de recherche français ou étrangers, des laboratoires publics ou privés. 
An International Journal at the Interface Between Chemistry and Physics

\title{
Effect of structural considerations on the development of free energy functionals for the square-well fluid
}

\author{
Thomas Bernet, Manuel M. Piñeiro, Frédéric Plantier \& Christelle Miqueu
}

To cite this article: Thomas Bernet, Manuel M. Piñeiro, Frédéric Plantier \& Christelle Miqueu (2018): Effect of structural considerations on the development of free energy functionals for the square-well fluid, Molecular Physics

To link to this article: https://doi.org/10.1080/00268976.2018.1438677

曲 Published online: 22 Feb 2018.

Submit your article to this journal [

Q View related articles 주

View Crossmark data 


\title{
Effect of structural considerations on the development of free energy functionals for the square-well fluid
}

\author{
Thomas Bernet ${ }^{\mathrm{a}}$, Manuel M. Piñeiro ${ }^{\mathrm{b}}$, Frédéric Plantier ${ }^{\mathrm{a}}$ and Christelle Miqueu $^{\mathrm{a}}$ \\ a Univ Pau \& Pays Adour/Total/CNRS, Laboratoire des Fluides Complexes et leurs Réservoirs - IPRA, UMR5150, 64600, Anglet, France; \\ ${ }^{\text {b}}$ Departamento de Física Aplicada, Facultad de Ciencias, Universidade de Vigo, Vigo, Spain
}

\begin{abstract}
In this work, we compare four inhomogeneous square-well fluid models, only distinct in the approximation on the pair correlation function present in the attractive free energy term of the density functional theory (DFT). Various criteria can be considered to select or validate an approximation, depending on fundamental interests or for a specified application. Here the considered criteria of selection were the verification of sum rules, and the prediction of both the adsorbed quantity and the density profiles. Even if a model satisfies one of these criteria, it may fail to describe others, so they can be considered supplementary to each other and in no case redundant. According to this study, one can attach more importance to any of these criteria depending on the specific objectives intended for the model development and purpose. They can serve as references in the development of new DFT models, eventually with other criteria, according to the objective of the work and the accuracy desired.
\end{abstract}

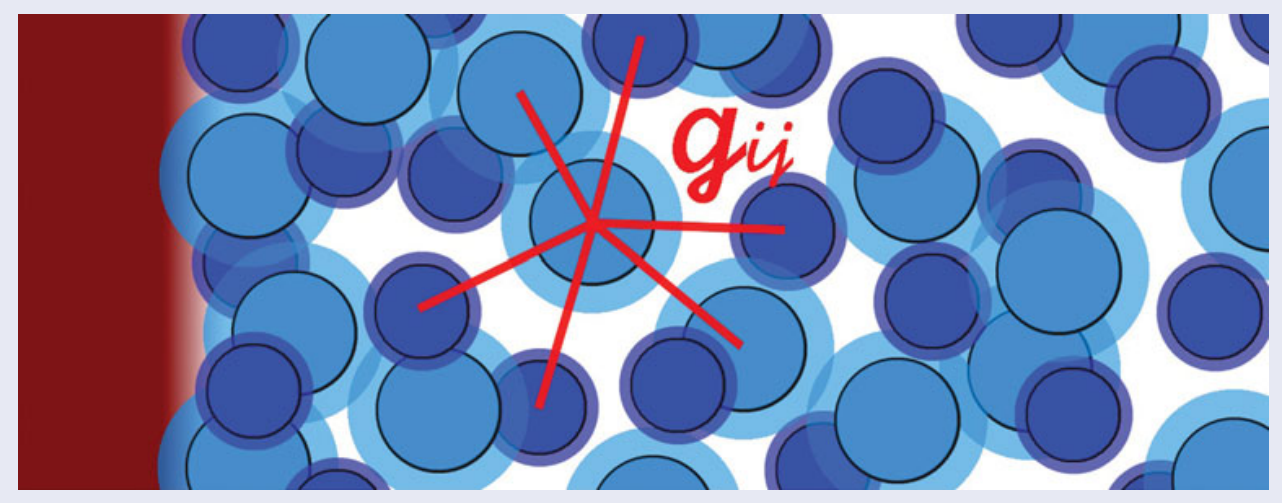

\section{ARTICLE HISTORY}

Received 20 December 2017 Accepted 5 February 2018

\section{KEYWORDS}

Density functional theory; fundamental-measure theory; pair correlation function; coarse-graining; mean-field

\section{Introduction}

The study of adsorption phenomena can be carried out with different fluid models, using various approximations within the same general theoretical framework such as the classical density functional theory (DFT) [1] considered in this work. This theory describes an inhomogeneous fluid through its free energy functional, which is the equivalent of the free energy for homogeneous fluids.

The free energy depends on functions which are the local densities of the chemical species constituting the fluid. The form of this functional depends on the microscopic representation assumed for the fluid, which contains intermolecular interactions that are repulsive at short range and attractive at longer distances. Moreover, the inhomogeneous fluid study requires different tools according to the variations of the fluid density. For vapour-liquid interfaces, as density gradients are rather smooth, local densities can be used to reproduce their usual thermodynamical properties. However, when fluid-solid interactions need to be considered, such as in the case of adsorption, density gradients are too sharply sloped to use the same representations and functions as for the case of fluid interfaces. In the DFT framework, this interaction can be considered as an external potential applied to the fluid (the considered system), but this does not indicate directly the dependence of the free energy functional to the density. This point allows considering

CONTACT Christelle Miqueu christelle.miqueu@univ-pau.fr

๑) 2018 Informa UK Limited, trading as Taylor \& Francis Group 
various approximations on the fluid representation. In particular, the weighted density approximations (WDA) allows not only taking into account the local density, but also the density of neighbouring points. To do so, one can consider a sphere centred on the specific point, delimiting the range of the effect of the neighbourhood. Thermodynamical properties are thus averaged from this sphere, and if the resulting approximation contains a pertinent physical sense to describe the system, it is thus possible to model the properties of the inhomogeneous fluid adequately.

This technique can be introduced by various ways, with different degrees of approximation, according to the averages applied or the weighting radius considered of the sphere. In practice, the choice of these approximations is related to the representation of the repulsive and attractive parts of the intermolecular potential, through the free energy functional. The repulsive part is generally described by the fundamental-measure theory (FMT) developed by Rosenfeld in 1989 [2]. This theory considers weightings on spheres of radii equal to the radii of individual hard-spheres. This model has shown very good results and many free energy functionals are now based on it, using the hard-sphere as a reference (for example, see [3-5]). Some of these functionals consider an additional attractive term, treated as a perturbation of the repulsive part. However, the description of this kind of terms often brings various approximations because of the lack of knowledge of the intermolecular potential. The coarse-grained (CG) method is one of the techniques similar to the FMT, as an example of WDA. In that case, the weighting is generally done with the interaction distance, defined with real or effective parameters, from the intermolecular interaction potential selected.

The addition of these approximations, and the choice of the terms for which they are applied, raises the question of the consistency of the model developed or used. In particular, some thermodynamical relations need to be satisfied by the model for the sake of consistency, between free energy, pressure, surface tension or pair correlation function. The latter is related to the probability to find a molecule at a certain distance of another. These relations are defined both for homogeneous and inhomogeneous phases, and a certain DFT may not satisfy all of them, according to its approximations. In particular, we need to consider approximations on the pair correlation function. Indeed, if we would know perfectly this function, we could characterise totally the system, which is of course not accessible.

The aim of this work is to compare these different approximations and their ability to satisfy various criteria. Among the countless approximations we can consider for this function, we will focus only on four of them. The first one is the mean-field (MF) approximation, which neglects interactions between molecules into the pair correlation function only, and which is used sometimes to describe long-range effects. The second one is based on expressions defined for the bulk phase. The third one is an application of the CG method. Finally, the fourth comes from the FMT. We could extend the benchmark to more functions, but we cannot be exhaustive, and these four cases already describe a range of quite different approximations within the same theoretical framework.

The question asked at the beginning of this work is to know for which terms we can apply these different approximations, and how. Then, the study of thermodynamical rules called sum rules [6] will be presented, as a first test of consistency and quality of these models. Finally, the study of the fluid microstructure predicted by each model will highlight other aspects of their performance. All these approximations will use the square-well potential to describe intermolecular interactions. This will constitute a common basis in order to clearly establish the importance of the pair correlation function used in the attractive contribution term of the free energy functional.

\section{Free energy functionals for the square-well fluid}

\subsection{DFT, weighted density approximations and hard-sphere description}

In the general classical DFT framework [1], the grand potential $\Omega$ is a functional of all the local densities $\rho_{i}(\vec{r})$, for the species $i$ at a given point $\vec{r}$ in the space. The relationship between this functional, the free energy functional $\mathcal{F}\left[\left\{\rho_{i}\right\}\right]$, an external potential $V_{\text {ext }, i}(\vec{r})$ and the chemical potential $\mu_{i}$ is

$$
\Omega\left[\left\{\rho_{i}\right\}\right]=\mathcal{F}\left[\left\{\rho_{i}\right\}\right]+\sum_{i} \int \rho_{i}(\vec{r})\left(V_{\text {ext }, i}(\vec{r})-\mu_{i}\right) \mathrm{d}^{3} r .
$$

The chemical potential $\mu_{i}$ is one of the homogeneous fluids. The system considered is the fluid, and the external potential $V_{\text {ext, } i}(\vec{r})$ takes into account the interaction between this fluid and a solid, in the case of adsorption. Because of the presence of this solid and the fluid-solid interaction, the structure of the fluid changes close to the interface. In real systems, attractive interactions between the fluid and the solid lead to an increase of the number of molecules close to the wall. But, for theoretical studies, where the goal is to obtain a good representation of the fluid itself, we can choose a repulsive external potential, as for instance a hard wall, to test the quality of the 
functional itself. We will consider this potential in further sections.

The description of the fluid is related to the free energy functional $\mathcal{F}\left[\left\{\rho_{i}\right\}\right]$. It is the equivalent of the equation of state for an inhomogeneous fluid. Like the grand potential, it is a functional of all the local densities $\rho_{i}(\vec{r})$, and it contains the fluid-fluid interactions. For homogeneous systems, this functional becomes the free energy function, and must be consistent with the chemical potential $\mu_{i}$ cited above. The combination of these elements leads to the description of the fluid at equilibrium, by the minimisation of the grand potential:

$$
\frac{\delta \Omega}{\delta \rho_{i}(\vec{r})}=\frac{\delta \mathcal{F}}{\delta \rho_{i}(\vec{r})}+V_{\mathrm{ext}, i}(\vec{r})-\mu_{i}=0
$$

The free energy functional is composed of an ideal term, describing the ideal gas contribution, and an excess term, containing the fluid-fluid interactions: $\mathcal{F}=\mathcal{F}^{\text {id }}+$ $\mathcal{F}^{e x}$. To obtain the density profile, associated with the microstructure of the fluid, this decomposition and the expression of the ideal free energy are used to obtain

$$
\rho_{i}(\vec{r})=\rho_{\text {bulk }, i} \exp \left(\beta \mu_{i}^{\mathrm{ex}}-\frac{\delta \beta \mathcal{F}^{\mathrm{ex}}}{\delta \rho_{i}(\vec{r})}-\beta V_{\mathrm{ext}, i}(\vec{r})\right) .
$$

The term $\frac{\delta \beta \mathcal{F}^{\mathrm{ex}}}{\delta \rho_{i}(\vec{r})}$ acts as an inhomogeneous chemical potential for the system, and it contains all the local densities $\left\{\rho_{i}(\vec{r})\right\}$. As it is done in DFT studies, this self-consistent equation is solved numerically, with the Picard iteration scheme (see for instance reference [6] for details).

To describe both the repulsive short-range and the attractive long-range interactions of the molecules, the potential between molecules $i$ and $j$ at distance $r$ is decomposed in two parts,

$$
u_{i j}(r)=u_{i j}^{\mathrm{HS}}(r)+u_{i j}^{\mathrm{att}}(r) .
$$

The hard-sphere potential (HS) corresponds to the repulsion and the notation att points out the attractive contribution. Because the hard-sphere repulsion determines to a great extent the fluid behaviour, we treat the attractive part as a perturbation of this potential. Otherwise, the Statistical Associating Fluid Theory for Variable Range of interaction (SAFT-VR [7]) decomposes the free energy into different contributions. For spherical molecules without association (the monomer term), the excess free energy is decomposed like the potential, $\mathcal{F}^{\mathrm{ex}}=\mathcal{F}^{\mathrm{HS}}+\mathcal{F}^{\text {att }}$. The term $\mathcal{F}^{\mathrm{HS}}$ describes the hardsphere contribution to the free energy and $\mathcal{F}^{\text {att }}$ is the attractive one. We will specify the attractive potential in the next section. First, we present the hard-sphere formalism, based on weighted functions.

WDAs are used into DFT to take into account the influence of neighbourhood at a certain point $\vec{r}$ of space. Indeed, the system is not correctly described with only local densities $\rho_{i}(\vec{r})$ into the functional $\mathcal{F}^{\mathrm{HS}}$ if the density variations are important. Thus, non-local DFT with weighted densities needs to be used. This kind of approximation leads to the coarse-grained (CG) model or the FMT, according to the weighting chosen (and other specific assumptions). In this work, we propose a general formalism associated with WDA, compatible both with coarse-grained models and the Kierlik and Rosinberg version of the FMT (KR-FMT) [8]. Contrarily to the original version of Rosenfeld [2], which contains scalar and vectorial weighted densities, the KR-FMT contains only scalar densities.

The weights depend on a sphere, characterised by a radius $\mathcal{R}$, which can correspond to the hard-sphere radius $R_{i}$, the diameter $\sigma_{i}=2 R_{i}$, the contact distance between two different hard-spheres $R_{i}+R_{j}$, or any other arbitrary distance, real or effective, according to the model. Weighting on a function, e.g. the local density $\rho_{i}(\vec{r})$, consists in doing averages of this function on the volume of the sphere $(\alpha=3)$, on the surface of the sphere $(\alpha=2)$ or on surface variations of the sphere $(\alpha=1,0)$. The corresponding weights use Heaviside function and its derivatives, as the Dirac distribution:

$$
\begin{aligned}
& p^{(3)}(\mathcal{R} ; r)=\Theta(\mathcal{R}-r) \\
& p^{(2)}(\mathcal{R} ; r)=\delta(\mathcal{R}-r) \\
& p^{(1)}(\mathcal{R} ; r)=\frac{1}{8 \pi} \delta^{\prime}(\mathcal{R}-r) \\
& p^{(0)}(\mathcal{R} ; r)=-\frac{1}{8 \pi} \delta^{\prime \prime}(\mathcal{R}-r)+\frac{1}{2 \pi r} \delta^{\prime}(\mathcal{R}-r)
\end{aligned}
$$

Applied to a general function $f$, the weighted function of type $\alpha$ can be written as

$$
n^{(\alpha)}(f ; \mathcal{R} ; \vec{r})=\int f\left(\vec{r}^{\prime}\right) p^{(\alpha)}\left(\mathcal{R} ;\left|\vec{r}^{\prime}-\vec{r}\right|\right) \mathrm{d}^{3} r^{\prime}
$$

(this notation was introduced in the work [9]). In the KRFMT, all of these weights are used to define weighted densities of a specie $i$ for the hard-sphere,

$$
n_{i}^{(\alpha)}(\vec{r})=n^{(\alpha)}\left(\rho_{i} ; R_{i} ; \vec{r}\right),
$$

and total weighted densities $n_{\alpha}(\vec{r})=\sum_{i} n_{i}^{(\alpha)}(\vec{r})$, with a sum over all the species. These latter are the functions of the free energy density functional $\Phi^{\mathrm{HS}}$ defined in the general FMT formalism. Different expressions of this functional are used, depending on the degree of approximation of the model. We use the White-Bear version mark 
II [10] of the FMT, with the functional

$$
\begin{aligned}
\Phi^{\mathrm{HS}}= & -\ln \left(1-n_{3}\right) n_{0}+\frac{1+\frac{1}{9} n_{3}{ }^{2}+\frac{1}{18} n_{3}{ }^{3}}{\left(1-n_{3}\right)} n_{1} n_{2} \\
& +\frac{1-\frac{4}{9} n_{3}+\frac{1}{18} n_{3}{ }^{2}}{24 \pi\left(1-n_{3}\right)^{2}} n_{2}{ }^{3} .
\end{aligned}
$$

From this quantity, we define the contribution of hardspheres at the free energy

$$
\beta \mathcal{F}^{\mathrm{HS}}=\int \Phi^{\mathrm{HS}}\left[\left\{n_{\alpha}(\vec{r})\right\}\right] \mathrm{d}^{3} r
$$

and the related inhomogeneous chemical potential for hard-spheres

$$
\begin{aligned}
\mu_{i}^{\mathrm{HS}}(\vec{r}) & =\frac{\delta \mathcal{F}^{\mathrm{HS}}}{\delta \rho_{i}(\vec{r})}=\left.\sum_{\alpha=0}^{3} \int \frac{\partial \Phi^{\mathrm{SD}}}{\partial n_{\alpha}}\right|_{\left(\vec{r}_{i}\right)} p^{(\alpha)}\left(R_{i} ;\left|\vec{r}_{i}-\vec{r}\right|\right) \mathrm{d}^{3} r_{i} \\
& =\sum_{\alpha=0}^{3} \mu_{i}^{(\alpha)}(\vec{r})
\end{aligned}
$$

with the weighted functions

$$
\mu_{i}^{(\alpha)}(\vec{r})=n^{(\alpha)}\left(\frac{\partial \Phi^{\mathrm{SD}}}{\partial n_{\alpha}} ; R_{i} ; \vec{r}\right) .
$$

Here, we use the general notation of weighted functions (6), applied on derivatives of the functional $\Phi^{\mathrm{HS}}$, with weights on a sphere of radius $R_{i}$. These weights come from the functional derivation of total weighted densities, w.r.t. the local density of the specie $i$,

$$
\frac{\delta n_{\alpha}\left(\vec{r}^{\prime}\right)}{\delta \rho_{i}(\vec{r})}=p^{(\alpha)}\left(R_{i} ;\left|\vec{r}-\vec{r}^{\prime}\right|\right) .
$$

\subsection{The square-well potential contribution to the attractive free energy functional}

The attractive contribution to the free energy functional, in the general DFT formalism [11] is given as a function of the attractive part of the potential $u_{i j}^{\text {att }}$ between two molecules of species $i$ and $j$, and the pair correlation function of these molecules $g_{i j}$,

$$
\begin{aligned}
& \beta \mathcal{F}^{\text {att }}=\frac{1}{2} \sum_{i, j} \iint \rho_{i}\left(\vec{r}_{i}\right) \rho_{j}\left(\vec{r}_{j}\right) g_{i j}\left(\vec{r}_{i} ; \vec{r}_{j}\right) \\
& \times \beta u_{i j}^{\text {att }}\left(\left|\vec{r}_{i}-\vec{r}_{j}\right|\right) \mathrm{d}^{3} r_{i} \mathrm{~d}^{3} r_{j} .
\end{aligned}
$$

Barker and Henderson [12] proposed to treat the pair correlation function of the (attractive) fluid as an expansion of the pair correlation function of the hard-sphere reference, $g_{i j}^{\mathrm{HS}}$; this last function being the first order of the expansion. The second order is computed by using the compressibility of hard-spheres $K^{\mathrm{SD}}$, the attractive part of the potential and the temperature with $\beta$. Barker and Henderson detailed the assumptions to write the pair correlation function as

$g_{i j}\left(\vec{r}_{i} ; \vec{r}_{j}\right)=\left(1-\frac{1}{2} K^{\mathrm{HS}} \beta u_{i j}^{\mathrm{att}}\left(\left|\vec{r}_{i}-\vec{r}_{j}\right|\right)\right) g_{i j}^{\mathrm{HS}}\left(\vec{r}_{i} ; \vec{r}_{j}\right)$,

and named this formulation, the macroscopic compressibility approximation. Then, they used another assumption to add a term to the second order, written $V\left(\frac{\partial g_{i j}^{\mathrm{HS}}}{\partial V}\right)$ for a homogeneous fluid, named the local compressibility approximation. Contrarily to our previous paper [9], we do not use this approximation in this work, because we will consider some further expressions for which this local description has no sense for inhomogeneous systems.

Now, the square-well potential is chosen for the attractive contribution between two molecules $i$ and $j$. This potential is defined by a constant depth $\varepsilon_{i j}$ for a distance included between the interaction distance of hardsphere $R_{i}+R_{j}$ and the attractive range $\lambda_{i} R_{i}+\lambda_{j} R_{j}$. Further, we will specify the well depth for interaction between two identical molecules $i$, written $\varepsilon_{i}$. According to the Lorentz-Berthelot mixing rule, the coupled well depth is $\varepsilon_{i j}=\sqrt{\varepsilon_{i} \varepsilon_{j}}$. Because it is attractive, the potential $u_{i j}^{\text {att }}(r)$ is negative, whereas the variable $\varepsilon_{i j}$ is positive. So, $u_{i j}^{\text {att }}(r)=$ $-\varepsilon_{i j} \times \varphi_{i j}(r)$ can be expressed as

$$
\varphi_{i j}(r)=\Theta\left(\lambda_{i} R_{i}+\lambda_{j} R_{j}-r\right)-\Theta\left(R_{i}+R_{j}-r\right)
$$

which defines the attractive zone of the potential. Seeing that $\left[\varphi_{i j}(r)\right]^{2}=\varphi_{i j}(r)$ we have

$$
\begin{aligned}
& \beta \mathcal{F}^{\mathrm{att}}=-\frac{1}{2} \sum_{i, j}\left(\beta \epsilon_{i j}\right) \iint \rho_{i}\left(\vec{r}_{i}\right) \rho_{j}\left(\vec{r}_{j}\right) g_{i j}\left(\vec{r}_{i} ; \vec{r}_{j}\right) \\
& \times \varphi_{i j}\left(\left|\vec{r}_{i}-\vec{r}_{j}\right|\right) \mathrm{d}^{3} r_{i} \mathrm{~d}^{3} r_{j} .
\end{aligned}
$$

using the value of the pair correlation function in the attractive zone, at the distance $\left|\vec{r}_{i}-\vec{r}_{j}\right|$ :

$$
g_{i j}^{\mathrm{att}}\left(\vec{r}_{i} ; \vec{r}_{j}\right)=\left(1+\frac{1}{2} K^{\mathrm{HS}} \beta \varepsilon_{i j}\right) g_{i j}^{\mathrm{HS}}\left(\vec{r}_{i} ; \vec{r}_{j}\right)
$$

The pair correlation function $g_{i j}^{\text {att }}$, thus defined, and its hard-sphere equivalent $g_{i j}^{\mathrm{HS}}$, depends on the two positions $\vec{r}_{i}$ and $\vec{r}_{j}$ of molecules $i$ and $j$. In the SAFT-VR approach [7], this dependence is changed to only one variable, by applying the mean-value theorem on $g_{i j}^{\mathrm{HS}}$, and considering the equivalent value at contact instead of the distance $\left|\vec{r}_{i}-\vec{r}_{j}\right|$. To do that, one needs to consider the effective 
density at contact, corresponding to the value of $g_{i j}^{\mathrm{HS}}$ at the real density, at the mean distance between two molecules. Then, the effective density is given by the real density and some coefficients, computed from an analytic approximated expression of the pair correlation function of the hard-sphere, as a function of the distance (see [13] for the function, and [7] for details of this method).

Because the function used to compute these coefficients is defined for an homogeneous fluid, an approximation is performed when using them in an inhomogeneous fluid formalism. However, one can assume that this approximation does not alter the main behaviour of the fluid microstructure, as shown in our previous publication [9] or with stronger but physical approximations (see the MF approximation below). Hence, in the following, we consider the pair correlation function at contact and at the effective density, $g_{i j, \text { eff }}^{\mathrm{HS}}$. If the variable of this function is chosen as $\vec{r}_{i}$, that means we apply the mean-value theorem on $\vec{r}_{j}$, which is replaced by the effective contact.

Thus, we obtain

$$
\begin{aligned}
\beta \mathcal{F}^{\mathrm{att}}= & -\frac{1}{2} \sum_{i, j}\left(\beta \varepsilon_{i j}\right) \int \rho_{i}\left(\vec{r}_{i}\right) g_{i j, \mathrm{eff}}^{\mathrm{att}}\left(\vec{r}_{i}\right) \\
& \times\left(\int \rho_{j}\left(\vec{r}_{j}\right) \varphi_{i j}\left(\left|\vec{r}_{i}-\vec{r}_{j}\right|\right) \mathrm{d}^{3} r_{j}\right) \mathrm{d}^{3} r_{i} .
\end{aligned}
$$

The dependence of $g_{i j, \text { eff }}^{\text {att }}\left(\vec{r}_{i}\right)$ will be specified below. Because the aim of this paper is to compare various approximations in this method, different approximated expressions of this function will be compared, according to the dependence on the densities.

In reference [9], we applied the FMT formalism to this functional, and derived it to compute the inhomogeneous chemical potential. In this work, the functional is first derived to define the attractive contribution of the chemical potential

$$
\mu_{k}^{\mathrm{att}}(\vec{r})=\frac{\delta \mathcal{F}^{\mathrm{att}}}{\delta \rho_{k}(\vec{r})}
$$

to obtain

$$
\begin{aligned}
\beta \mu_{k}^{\text {att }}(\vec{r})= & -\sum_{i}\left(\beta \varepsilon_{i k}\right) g_{i k, \text { aff }}^{\text {att }}(\vec{r}) \int \rho_{i}\left(\vec{r}_{i}\right) \varphi_{i k}\left(\left|\vec{r}_{i}-\vec{r}\right|\right) \mathrm{d}^{3} r_{i} \\
& -\frac{1}{2} \sum_{i, j}\left(\beta \varepsilon_{i j}\right) \int \rho_{i}\left(\vec{r}_{i}\right) \frac{\delta g_{i j, \text { eff }}^{\text {att }}\left(\vec{r}_{i}\right)}{\delta \rho_{k}(\vec{r})} \\
& \times\left(\int \rho_{j}\left(\vec{r}_{j}\right) \varphi_{i j}\left(\left|\vec{r}_{i}-\vec{r}_{j}\right|\right) \mathrm{d}^{3} r_{j}\right) \mathrm{d}^{3} r_{i}
\end{aligned}
$$

The functional derivative $\frac{\delta g_{i j, \text { eff }}^{\text {att }}\left(\vec{r}_{i}\right)}{\delta \rho_{k}(\vec{r})}$ will be computed for each approximation presented in the next section. To precise it, we need to choose an approximation on its dependence on the local density $\rho_{k}(\vec{r})$, through bulk or weighted densities into $g_{i j, \text { eff }}^{\text {att }}\left(\vec{r}_{i}\right)$.

Here, we apply the general notation for weighting to the integrals above

$$
\int \rho_{i}\left(\vec{r}_{i}\right) \varphi_{i k}\left(\left|\overrightarrow{r_{i}}-\vec{r}\right|\right) \mathrm{d}^{3} r_{i}=\bar{v}_{i k}^{(3)}(\vec{r})-\bar{n}_{i k}^{(3)}(\vec{r}) .
$$

Thus, we apply averages on the volume of a sphere of radius $R_{i}+R_{j}$, with

$$
\bar{n}_{i j}^{(\alpha)}(\vec{r})=n^{(\alpha)}\left(\rho_{i} ; R_{i}+R_{j} ; \vec{r}\right),
$$

and averages on the volume of a sphere of radius $\lambda_{i} R_{i}+$ $\lambda_{j} R_{j}$, with

$$
\bar{v}_{i j}^{(\alpha)}(\vec{r})=n^{(\alpha)}\left(\rho_{i} ; \lambda_{i} R_{i}+\lambda_{j} R_{j} ; \vec{r}\right) .
$$

The differences in Equations (22) and (23) are related to the attractive zone on the potential, centered in the point $\vec{r}$. In this approach, it is not necessary to consider the FMT decomposition of these two spheres, because at the end, only averages are computed on the volume of these spheres, thus describing an interaction and not individual spheres, as in the hard-sphere description.

At the end,

$$
\begin{aligned}
\beta \mu_{k}^{\mathrm{att}}(\vec{r})= & -\sum_{i}\left(\beta \varepsilon_{i k}\right) g_{i k, \mathrm{eff}}^{\mathrm{att}}(\vec{r})\left(\bar{v}_{i k}^{(3)}(\vec{r})-\bar{n}_{i k}^{(3)}(\vec{r})\right) \\
& -\frac{1}{2} \sum_{i, j}\left(\beta \varepsilon_{i j}\right) \int \rho_{i}\left(\vec{r}_{i}\right) \frac{\delta g_{i j, \mathrm{eff}}^{\mathrm{att}}\left(\vec{r}_{i}\right)}{\delta \rho_{k}(\vec{r})} \\
& \times\left(\bar{v}_{j i}^{(3)}\left(\vec{r}_{i}\right)-\bar{n}_{j i}^{(3)}\left(\vec{r}_{i}\right)\right) \mathrm{d}^{3} r_{i} .
\end{aligned}
$$

In order to obtain density profiles, one needs to choose a model for $g_{i j \text {,eff }}^{\text {att }}(\vec{r})$ and its derivative, in order to compute the inhomogeneous chemical potential $\mu_{k}^{\text {att }}(\vec{r})$, and use the result in the Picard iteration algorithm.

\subsection{List of approximations on the effective pair correlation function}

\subsubsection{The mean-field approximation}

The strongest approximation one can consider on the effective pair correlation function $g_{i j \text {, eff }}^{\text {att }}(\vec{r})$ that does not eliminate the attractive term is the $M F$ approximation. It is worth noting here that this approximation is only applied for the pair correlation function in the general framework defined in the previous section. Other models considering a MF approximation can present other results because they use another general framework.

The hypothesis used here consists in neglecting the correlations into this function $g_{i j \text {, eff }}^{\text {att }}(\vec{r})$, used into the 
attractive free energy, and thus neglecting the BarkerHenderson expansion. That means we use

$$
\left.g_{i j, \mathrm{eff}}^{\mathrm{att}}\right|_{\mathrm{MF}}=1
$$

The consequence of this approach is that all the inhomogeneous aspects are contained into the weighted densities $\left(\bar{v}_{i j}^{(3)}(\vec{r})-\bar{n}_{i j}^{(3)}(\vec{r})\right)$, from the expression of the attractive part of the potential $u_{i j}^{\text {att }}$ with the weighted density formalism. This constitutes a first model, which has presented good results on adsorption studies, even if its main hypothesis is strong (see [14] for recent considerations).

Equation (24) is now written in a really simple form, because of the cancellation of the derivative of $g_{i j, \text { eff }}^{\text {att }}(\vec{r})$ :

$$
\left.\beta \mu_{k}^{\mathrm{att}}(\vec{r})\right|_{\mathrm{MF}}=-\sum_{i}\left(\beta \varepsilon_{i k}\right)\left(\bar{v}_{i k}^{(3)}(\vec{r})-\bar{n}_{i k}^{(3)}(\vec{r})\right)
$$

With the aim to verify whether this approximation is too strong for a correct description of the adsorption, we will compute density profiles with this functional and compare them to simulation results for the same system. Before this, we present other approximations on the function $g_{i j \text {,eff }}^{\text {att }}(\vec{r})$. Several approaches exist describing the pair correlation function beyond the MF approximation for various kinds of inhomogeneous systems (see the recent reference [15] with nematic liquid crystals for example). A common
With the White-Bear II version of the FMT [10], the corresponding bulk effective pair correlation function is

$$
\begin{aligned}
\left.g_{i j, \mathrm{eff}}^{\mathrm{HS}}\right|_{\mathrm{Bulk}}= & \frac{1}{1-\xi_{i j}^{\mathrm{eff}}}+\left[\frac{R_{i} R_{j}}{R_{i}+R_{j}} \frac{\xi_{2}}{3 \xi_{3}}\right] \frac{3 \xi_{i j}^{\mathrm{eff}}+\left(\xi_{i j}^{\mathrm{eff}}\right)^{2}}{\left(1-\xi_{i j}^{\mathrm{eff}}\right)^{2}} \\
& +\left[\frac{R_{i} R_{j}}{R_{i}+R_{j}} \frac{\xi_{2}}{3 \xi_{3}}\right]^{2} \frac{2\left(\xi_{i j}^{\mathrm{eff}}\right)^{3}}{\left(1-\xi_{i j}^{\mathrm{eff}}\right)^{3}}
\end{aligned}
$$

with $\xi_{i j}^{\text {eff }}=c_{1}\left(\lambda_{i j}\right) \xi_{3}+c_{2}\left(\lambda_{i j}\right) \xi_{3}{ }^{2}+c_{3}\left(\lambda_{i j}\right) \xi_{3}{ }^{3}$ and the SAFT-VR coefficients [7]

$$
\left(\begin{array}{l}
c_{1} \\
c_{2} \\
c_{3}
\end{array}\right)=\left(\begin{array}{ccc}
2.25855 & -1.50349 & 0.249434 \\
-0.669270 & 1.40049 & -0.827739 \\
10.1576 & -15.0427 & 5.30827
\end{array}\right)\left(\begin{array}{c}
1 \\
\lambda_{i j} \\
\lambda_{i j}{ }^{2}
\end{array}\right)
$$

where $\lambda_{i j}$ is the coupled attractive range parameter $\lambda_{i j}=$ $\frac{\lambda_{i} R_{i}+\lambda_{j} R_{j}}{R_{i}+R_{j}}$.

The attractive term of the free energy with this expression of the pair correlation function is proportional to the MF result. However, because $g_{i j \text {,eff }}^{\text {HS }}$ contains the bulk densities here, its functional derivative is not zero and the chemical potential of the attractive term is not proportional to the MF one.

To compute the compressibility used in the BarkerHenderson expansion, we use the expression related to the White-Bear II version of the FMT (computed here [9]),

$$
\left.K^{\mathrm{HS}}\right|_{\text {Bulk }}=\frac{\left(1-\xi_{3}\right)^{4} \xi_{0}}{\left(1-\xi_{3}\right)^{2} \xi_{0}+\left(2+\frac{4}{3} \xi_{3}{ }^{2}-\frac{2}{3} \xi_{3}{ }^{3}\right)\left(1-\xi_{3}\right) \xi_{1} \xi_{2}+\left(9-8 \xi_{3}+7 \xi_{3}{ }^{2}-2 \xi_{3}{ }^{3}\right) \frac{1}{36 \pi} \xi_{2}{ }^{3}} .
$$

approach consists in considering the MF approximation as a long-range effect on correlations, and add another term to describe short-range effects. This leads to add terms depending on the pair correlation function at contact (see references [16] and [17] as applications of this technique to Local DFT, and reference [3] to Non-Local DFT). We do not test this method in this work, in order to use only the SAFT-VR treatment, with effective densities, in the three other approximations below.

\subsubsection{The Bulk approximation}

The effective pair correlation function considered in this section is the bulk one. This approach was developed in our previous work [9] and rests upon the idea of using the bulk SAFT-VR treatment only on the bulk pair correlation function, and use this homogeneous result directly into the functional. $g_{i j \text {,eff }}^{\mathrm{HS}}\left[\left\{\xi_{\alpha}\right\}\right]$ is then given as a function of the Scaled Particle Theory variables $\left\{\xi_{\alpha}\right\}$ that are the bulk limit of the weighted densities for a hard-sphere $\left\{n_{\alpha}(\vec{r})\right\}$.
Then, Equation (24) becomes

$$
\begin{aligned}
\left.\beta \mu_{k}^{\text {att }}(\vec{r})\right|_{\text {Bulk }}= & -\sum_{i}\left(\beta \varepsilon_{i k}\right) g_{i k, \text { eff }}^{\text {att }}(\vec{r})\left(\bar{v}_{i k}^{(3)}(\vec{r})-\bar{n}_{i k}^{(3)}(\vec{r})\right) \\
& -\frac{1}{2} \sum_{i, j}\left(\beta \varepsilon_{i j}\right) \frac{\partial g_{i j, \mathrm{eff}}^{\text {att }}}{\partial N_{k}} \\
& \times \int \rho_{i}\left(\vec{r}_{i}\right)\left(\bar{v}_{j i}^{(3)}\left(\vec{r}_{i}\right)-\bar{n}_{j i}^{(3)}\left(\vec{r}_{i}\right)\right) \mathrm{d}^{3} r_{i} .
\end{aligned}
$$

One can obtain Equation (30) with the functional described in reference [9], but here, $g_{i j, \text { eff }}^{\text {att }}$ does not contain the term due to the local compressibility approximation. There is also a difference of computation between the treatment proposed here and the previous one [9], because here we apply the weighted function formalism directly on the chemical potential, and not on the functional itself. Here, we do not decompose the expressions of the first and second order of the expansion of the attractive term; we consider only the resultant attractive contribution. 


\subsubsection{The coarse-grained approximation}

In this approximation, we assume that the effective pair correlation function $g_{i j \text {,eff }}^{\text {att }}$ depends on the spatial position $\vec{r}$. In local DFT, this dependence is through the local densities $\left\{\rho_{i}(\vec{r})\right\}$, and in non-local DFT through the weighted densities. The coarse-grained (CG) approximation into DFT was successfully used in various adsorption studies (for example, see [3,4]), from Percus works [18]. This approach considers generally a weighting on the volume of a sphere of radius $\sigma_{i}$. This distance corresponds to the interaction distance between two identical hard-spheres. This way, this method is well adapted to describe pure fluids only. To write a functional for an inhomogeneous fluid, we use the equivalent function for the homogeneous limit, and we replace the variable $\rho_{\text {bulk, } i}$ by the function $\bar{\rho}_{i}(\vec{r})$, which is

$$
\bar{\rho}_{i}(\vec{r})=\frac{\bar{n}_{i i}^{(3)}(\vec{r})}{\frac{4 \pi}{3}\left(2 R_{i}\right)^{3}},
$$

using the weighted density (22). This weighting is not fully adapted to describe mixtures, because the weighting radius is $\sigma_{i}=2 R_{i}$ for a specie $i$ only, instead of $R_{i}+R_{j}$, that could take into account different species $i$ and $j$. Moreover, the packing fraction $\xi_{3}=\sum_{i} \frac{4 \pi}{3} \rho_{i} R_{i}^{3}$ becomes $\eta(\vec{r})=$ $\sum_{i} \frac{4 \pi}{3} \bar{\rho}_{i}(\vec{r}) R_{i}{ }^{3}$. Using this function $\eta(\vec{r})$ for an inhomogeneous mixture is another approximation, because the weighting considers only the interaction distance $2 R_{i}$.

For the hard-sphere system, the idea of replacing this volumetric weighting on interaction distance by weightings on individual spheres conduced Rosenfeld [2] to decompose the function $\Theta\left(R_{i}+R_{j}-r\right)$. The resulting FMT framework is then a WDA for mixtures, using separated weights $R_{i}$ and $R_{j}$. For hard-sphere systems, the FMT free energy yields better results than most of the CG versions [19].

The hard-sphere contribution to the inhomogeneous chemical potential has been treated with the FMT. Then, the attractive part of the potential $u_{i j}^{\text {att }}$ has been treated with the general WDA formalism defined at the beginning. We assume that the main feature of density profiles comes from these treatments, and here, we apply the CG approximation on the pair correlation function only. With this approach, the aim is to limit the additional approximation for mixtures, as this CG approximation is not applied on all the terms of the free energy functional.

Then, the effective pair correlation function (from the Carnahan-Starling [20] equation of state) is

$$
\left.g_{i j, \mathrm{eff}}^{\mathrm{HS}}\right|_{C G}=\frac{1-\frac{1}{2} \eta_{i j}^{\mathrm{eff}}}{\left(1-\eta_{i j}^{\mathrm{eff}}\right)^{3}}
$$

with the weighted effective density $\eta_{i j}^{\text {eff }}=c_{1}\left(\lambda_{i j}\right) \eta+$ $c_{2}\left(\lambda_{i j}\right) \eta^{2}+c_{3}\left(\lambda_{i j}\right) \eta^{3}$. The coefficients $c_{k},(k=1,2,3)$ are the same as the ones in the precedent section. Moreover, the compressibility is now

$$
\left.K^{\mathrm{HS}}\right|_{\mathrm{CG}}=\frac{(1-\eta)^{4}}{1+4 \eta+4 \eta^{2}-4 \eta^{3}+\eta^{4}} .
$$

In this CG approximation, $g_{i j, \text { eff }}^{\mathrm{HS}}[\eta(\vec{r})]$ leads to the inhomogeneous chemical potential

$$
\begin{aligned}
\left.\beta \mu_{k}^{\mathrm{att}}(\vec{r})\right|_{\mathrm{CG}}= & -\sum_{i}\left(\beta \varepsilon_{i k}\right) g_{i k, \mathrm{eff}}^{\mathrm{att}}(\vec{r})\left(\bar{v}_{i k}^{(3)}(\vec{r})-\bar{n}_{i k}^{(3)}(\vec{r})\right) \\
& -\frac{1}{2} \sum_{i, j}\left(\beta \varepsilon_{i j}\right) Y_{i j k}^{(3)}(\vec{r})
\end{aligned}
$$

with the weighted function

$$
Y_{i j k}^{(\alpha)}(\vec{r})=n^{(\alpha)}\left(\rho_{i} \frac{\partial g_{i j, \text { eff }}^{\text {att }}}{\partial \eta}\left(\bar{v}_{j i}^{(3)}-\bar{n}_{j i}^{(3)}\right) ; 2 R_{k} ; \vec{r}\right) .
$$

This function is obtained with the same methodology used with the hard-sphere chemical potential, at the end of Section 2.1, for which the weighted function is not the density. The derivation of $\eta(\vec{r})$ leads to the weighting radius $2 R_{k}$, and because $\eta$ depends only on a weighting on the volume, we have to consider only $\alpha=3$ for $Y_{i j k}^{(\alpha)}(\vec{r})$.

\subsubsection{The KR-FMT approximation}

Other weightings into the effective pair correlation function are conceivable. If one chooses those of the FMT, $g_{i j \text {,eff }}^{\mathrm{HS}}\left[n_{\alpha}(\vec{r})\right]$ must be developed. This expression depends on the version of the FMT. The original Rosenfeld formulation [2] contains scalar and vectorial weighted functions. Next, Kierlik and Rosinberg [8] proposed another version for the hard-sphere (noted KR-FMT), equivalent to the Rosenfeld's one (only for the Scaled-Particle Theory reference [21]), with only scalar weighted densities. The relation between the scalar and the vectorial contributions in the free energy density led $\mathrm{Yu}$ and $\mathrm{Wu}$ [22] to include a vectorial term into their version of the pair correlation function, following Rosenfeld's formulation of the FMT. In the KR-FMT approximation, the use of this correction is not justified, and so, another pair correlation function must be developed.

To do so, we have changed the variables $\xi_{3}, \xi_{2}$ and $\xi_{i j}^{\text {eff }}$ from the bulk expression (27) by $n_{3}(\vec{r}), n_{2}(\vec{r})$ and $n_{i j}^{\text {eff }}(\vec{r})$ and obtained

$$
\left.g_{i j, \mathrm{eff}}^{\mathrm{HS}}\right|_{\mathrm{KR}-\mathrm{FMT}}=\frac{1}{1-n_{i j}^{\mathrm{eff}}}+\left[\frac{R_{i} R_{j}}{R_{i}+R_{j}} \frac{n_{2}}{3 n_{3}}\right] \frac{3 n_{i j}^{\mathrm{eff}}+\left(n_{i j}^{\mathrm{eff}}\right)^{2}}{\left(1-n_{i j}^{\mathrm{eff}}\right)^{2}}
$$




$$
+\left[\frac{R_{i} R_{j}}{R_{i}+R_{j}} \frac{n_{2}}{3 n_{3}}\right]^{2} \frac{2\left(n_{i j}^{\mathrm{eff}}\right)^{3}}{\left(1-n_{i j}^{\mathrm{eff}}\right)^{3}}
$$

with $n_{i j}^{\text {eff }}=c_{1}\left(\lambda_{i j}\right) n_{3}+c_{2}\left(\lambda_{i j}\right) n_{3}{ }^{2}+c_{3}\left(\lambda_{i j}\right) n_{3}{ }^{3}$. Likewise, we consider $K^{\mathrm{HS}}\left[\left\{n_{\alpha}(\vec{r})\right\}\right]$ from its bulk expression (29), obtained with the White-Bear II FMT.

The compact expression resulting for the chemical potential is

$$
\begin{aligned}
\left.\beta \mu_{k}^{\text {att }}(\vec{r})\right|_{\mathrm{KR}-\mathrm{FMT}}= & -\sum_{i}\left(\beta \varepsilon_{i k}\right) g_{i k, \mathrm{eff}}^{\mathrm{att}}(\vec{r})\left(\bar{v}_{i k}^{(3)}(\vec{r})-\bar{n}_{i k}^{(3)}(\vec{r})\right) \\
& -\frac{1}{2} \sum_{i, j}\left(\beta \varepsilon_{i j}\right) \sum_{\alpha=0}^{3} Z_{i j k}^{(\alpha)}(\vec{r})
\end{aligned}
$$

with the weighted function

$$
Z_{i j k}^{(\alpha)}(\vec{r})=n^{(\alpha)}\left(\rho_{i} \frac{\partial g_{i j, \text { eff }}^{\text {att }}}{\partial n_{\alpha}}\left(\bar{v}_{j i}^{(3)}-\bar{n}_{j i}^{(3)}\right) ; R_{k} ; \vec{r}\right) .
$$

Here, all the weighting $(\alpha=3,2,1,0)$ are considered with $Z_{i j k}^{(\alpha)}(\vec{r})$ to compute $\mu_{k}^{\text {att }}(\vec{r})$. Then, we notice that the functional $g_{i j \text {,eff }}^{\mathrm{HS}}$ of $\left\{n_{\alpha}(\vec{r})\right\}$ is derived and leads to the weighting radius $R_{k}$ into $Z_{i j k}^{(\alpha)}(\vec{r})$.

Another point has to be highlighted. The hard-sphere pair correlation function must be consistent with the free energy density for hard-spheres (such as it must be consistent with the pressure). The different approximations presented should not satisfy this consistency in the same way. The study of the sum rules in the following approaches some aspects of this question.

\section{Sum rules}

Sum rules establish relations between the inhomogeneous fluid microstructure, through the density profiles, and thermodynamical properties, such as pressure or surface tension. To check if these rules are verified, the relation between an inhomogeneous phase near a wall and a bulk homogeneous phase, at the packing fraction $\eta=$ $\frac{4 \pi}{3} \rho_{\text {bulk }} R^{3}$ has been considered.

To do so, contrarily to the hard-sphere fluid, various parameters of a square-well fluid, related to the attractive part, must be specified. This way, the following relative errors on sum rules are quantitative only for a particular square-well fluid, but the global study informs about general tendencies of the different approximations evoked. Indeed, the orders of magnitude of the squarewell fluid parameters are chosen to correspond to those of the SAFT-VR equation [7] (for the monomer contribution of molecules in standard conditions). For the study of a pure square-well fluid, $k_{B} T / \varepsilon=2$ and $\lambda=1.5$ were respectively chosen for the well depth and the attractive range.

We have applied to the square-well fluid the approach and definitions detailed in the Roth's review of FMT [6] for hard-spheres. The thermodynamic quantities are defined for one planar wall, normal to the axis $z$. Computations of density profiles are done by considering one hard wall at $z=0$, describing a position which is independent of the size of the molecules. The accessible positions of the center of a molecule of radius $R_{i}$ are for $z \geq R_{i}{ }^{+}$. Finally, thermodynamic quantities relative to the adsorption are presented below for one wall. To do so, the distance $L_{z}$ is defined as the normal distance from the left wall to a point in the bulk phase. Thus, this distance will be used in definitions of thermodynamic quantities relative to only one wall.

\subsection{Contact theorem}

The contact theorem traduces the mechanical equilibrium between the fluid and the wall through thermodynamic properties. Considering only one hard wall, the contact theorem establishes the relation between the bulk pressure $P$ and local densities at contact $\rho_{i}\left(z=R_{i}^{+}\right)$against the wall. For a planar hard wall, the theorem is simplified to

$$
\beta P=\sum_{i} \rho_{i}\left(z=R_{i}^{+}\right),
$$

because the summed quantity is reduced to the density at contact.

By solving Equation (3) for the four different approximations of the pair correlation function presented, we obtain different density profiles and different values of the density at contact against the wall. Moreover, pressure is computed with the equation of state of each model, obtained from the bulk limit of the free energy. Then, the validation of the sum rule shows an internal consistency of the theory and its approximations. It does not inform if the absolute computed values $\beta P$ and $\sum_{i} \rho_{i}\left(z=R_{i}{ }^{+}\right)$are correct or not, because here, they are not compared with simulation data (see the following sections for comparisons). Here, the comparison is relative and we represent the relative error between the summed quantity $\Sigma_{i} \rho_{i}(z=$ $R_{i}^{+}$) and the thermodynamical quantity $\beta P$,

$$
\operatorname{err}_{\text {contact }}=\frac{\beta P-\sum_{i} \rho_{i}\left(z=R_{i}^{+}\right)}{\beta P} .
$$

Relative errors on the contact theorem with the different approximations are presented in Figure 1. With the MF approximation, the relative error is zero, considering 


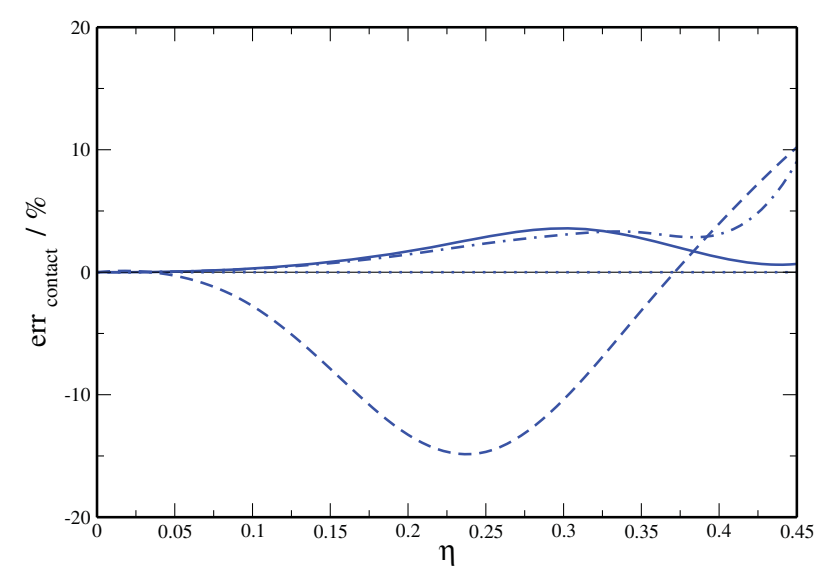

Figure 1. Relative errors on the contact theorem of the pure square-well fluid studied. Dotted, dash, dash-dotted and solid lines represent, respectively, the result for MF, bulk, CG and KR-FMT approximations.

the numerical precision of the computations, which satisfies the sum rule. With the CG and the KR-FMT pair correlation function, the error is not zero, but is still inferior to $3.5 \%$, at $\eta<0.4$. At higher densities, the error with the KR-FMT approximation decreases, contrary to the CG one. Except in this last case, we consider that these approximations as a whole are acceptable. On the other hand, the Bulk approximation for the pair correlation function presents important values for err $_{\text {contact }}$ (up to $15 \%$ ), indicating that it does not satisfy the contact theorem at all.

\subsection{Gibbs adsorption theorem}

The Gibbs adsorption theorem establishes the relation between the excess adsorbed quantity (relative to the bulk one) and the variation of the surface tension w.r.t. the bulk chemical potential. The relation corresponds to

$$
-\left(\frac{\mathrm{d} \gamma}{\mathrm{d} \mu_{i}}\right)_{T, V}=\int\left(\rho_{i}(z)-\rho_{\text {bulk }, i}\right) \mathrm{d} z .
$$

where $A$ stands for the area related to the surface of the wall, and $V$ is the volume against this wall, defined with the normal distance $L_{z}$, as $L_{z}=V / A$. With this notation, the surface tension (for one wall) is computed from $\gamma=$ $(\Omega+P V) / A$, with Equation (1) for the grand potential $\Omega$. For a hard planar wall, in one dimension, the surface tension becomes

$$
\gamma=\mathcal{F}_{1 D}-\sum_{i} \mu_{i} \int_{0}^{L_{z}} \rho_{i}(z) \mathrm{d} z+P L_{z}
$$

where $\mathcal{F}_{1 D}=\mathcal{F} / A$ is the one-dimensional free energy.

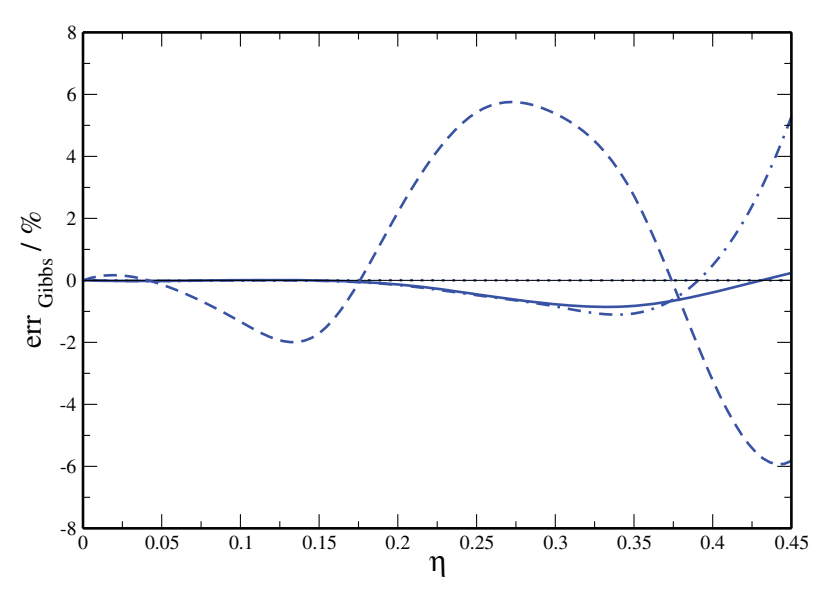

Figure 2. Relative errors on the Gibbs adsorption theorem of the pure square-well fluid studied.

As for the first sum rule, we consider the relative error between the summed quantity $\int\left(\rho_{i}(z)-\rho_{\text {bulk, } i}\right) \mathrm{d} z$ and the thermodynamic quantity $-\left(\frac{\mathrm{d} \gamma}{\mathrm{d} \mu_{i}}\right)_{T, V}$,

$$
\operatorname{err}_{\text {Gibbs }}=\frac{-\left(\frac{\mathrm{d} \gamma}{\mathrm{d} \mu_{i}}\right)_{T, V}-\int\left(\rho_{i}(z)-\rho_{\text {bulk }, i}\right) \mathrm{d} z}{-\left(\frac{\mathrm{d} \gamma}{\mathrm{d} \mu_{i}}\right)_{T, V}}
$$

Relative errors on the Gibbs adsorption theorem with the different approximations are presented in Figure 2. err $\mathrm{Gibbs}_{\text {is }}$ zero with the MF approximation, considering the numerical precision of the computations. For other approximations, the global conclusions are the same than those of the first sum rule: with the Bulk pair correlation function we observe significant and irregular $\operatorname{err}_{\text {Gibbs }}$ (up to $6 \%$ ); with the CG approximation the relative error is small for $\eta<0.4$ (less than 1\%) and increases at higher densities; with the KR-FMT approximation the difference is less than $1 \%$ for all densities. When the pair correlation function is different from 1 , the use of various approximations (Barker-Henderson expansion and weights) leads to different adsorbed quantities, according to the route (1.h.s. or r.h.s. term of the Equation (41)), and the sum rule is not fully satisfied.

This highlights the importance of the consistency between the (effective) pair correlation function $g_{i j \text {,eff' }}^{\mathrm{HS}}$ the free energy density $\Phi^{\mathrm{SD}}$ and the bulk pressure $P$. However, the MF approximation satisfies the sum rule, whereas the consistency between $\Phi^{\mathrm{SD}}$ or $P$ with the function $g_{i j \text {, eff }}^{\mathrm{HS}}=1$ is nonexistent in this case. We interpret this point by considering that the sum rule does not test directly and totally the consistency between these functions. It only shows a consistency between the density profile and the derivative of the surface tension w.r.t. the bulk chemical potential, computed from the same $g_{i j \text {,eff }}^{\mathrm{HS}}$ 


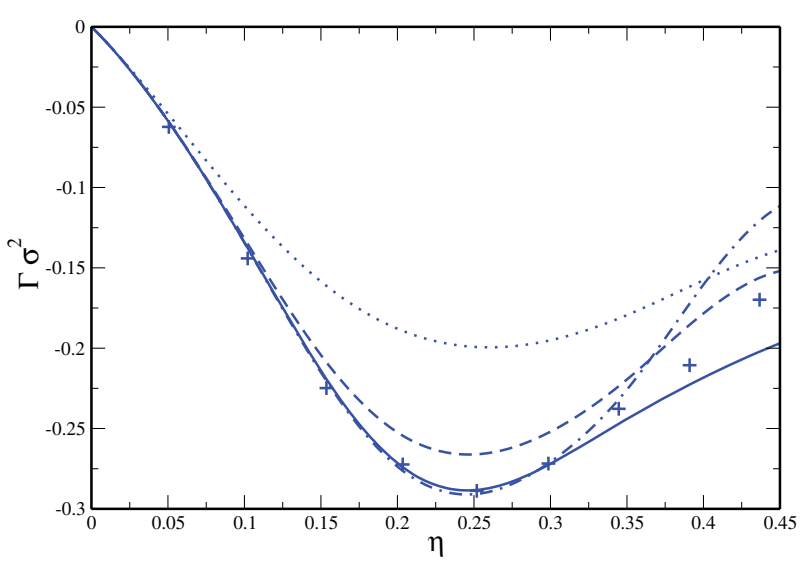

Figure 3. Excess adsorption quantity of the pure square-well fluid studied.

i.e. with the same degree of approximation. Here, the validation of the sum rule does not correspond to a higher consistency between $g_{i j \text {,eff }}^{\mathrm{HS}}$ and $\Phi^{\mathrm{SD}}$, compared to other models, but corresponds to a stronger approximation.

According to the choice of the pair correlation function, the error on the sum rule is acceptable or not. Another way to show the importance of these degrees of approximation is to compute the excess adsorbed quantity (absolute, and not only related to the Gibbs sum rule). Because of the definition of $L_{z}$, the surface tension is positive and the excess adsorption is negative (see reference [6] for details). In Figure 3, we represent the excess adsorption computed with the integral formulation, both for theory and simulation,

$$
\Gamma_{i}=\int\left(\rho_{i}(z)-\rho_{\mathrm{bulk}, i}\right) \mathrm{d} z
$$

This figure clearly shows that the excess adsorption is strongly underestimated (in absolute value) with the MF approximation, even at low densities. As considered above, even if the sum rules are satisfied in this case, the approximation is too strong to respect some other thermodynamic considerations. With the Bulk approximation, the excess adsorbed quantity is underestimated, but much less than for the MF approximation. However, CG and KR-FMT approximations present the same values for $\eta<0.3$, with a very good agreement with the simulations. At higher densities, the CG approximation underestimates the excess adsorption quantity, whereas the KRFMT approximation overestimates it.

If there is a difference between the adsorbed quantity predicted by the theory and the simulation, there is also a difference between the corresponding density profiles, used in Equation (44). But on the contrary, two different profiles can lead to the same adsorbed quantity, so one has to check the density profiles as well to obtain more information on these square-well fluid microstructures.

\section{Density profiles}

\subsection{Density profiles of a pure square-well fluid}

We represent density profiles at low, medium and high densities (respectively at $\eta=0.15,0.30$ and 0.45 ) for the four approximations compared in Section 3 . The squarewell pure fluid parameters are fixed as before and only a hard planar wall is placed at $z=0$. For these systems, the density profiles are compared to new NVT (i.e. at constant Number of particules, Volume and Temperature) Monte Carlo simulations computed at the same thermodynamical conditions.

Figure 4 represents the density profiles at $\eta=0.15$, 0.30 and 0.45 , respectively. The MF approximation overestimates densities at each point, in particular near the wall. While simulation and other models show a density smaller than the bulk density at $\eta=0.15$ and $z<1.5 \sigma$, the MF approximation leads to positive values, such as at higher densities. High value of density at the contact distance with the wall (at $z=0.5 \sigma$ ) is the typical behaviour of the purely repulsive hard-sphere fluid. With an attractive part in the potential, a difference is observed at low densities: the attraction between fluid molecules has then a prevailing influence on the fluid microstructure. At higher densities, more molecules are pushed against the hard wall, and this behaviour then is imposed over the attraction between molecules. The resulting profiles are closer to the hard-sphere fluid ones in this case.

At $\eta=0.15$ (Figure 4(a)), as shown in the reference [9], the main behaviour of the density profiles is recovered with the Bulk approximation on the pair correlation function, with a good agreement with simulation results. However, small deviations are observed close to the wall, contrarily to the profiles obtained with the CG and the KR-FMT approximations, which present an excellent agreement with simulation at any distance from the wall. At $\eta=0.3$ (Figure 4(b)), secondary layers are a bit displaced and their amplitudes change also according to the approximation made. The microstructures are similar, with a convergence to the bulk density far from the wall. The models are qualitatively good, even if some small differences appear with simulation results.

However, at $\eta=0.45$ (Figure 4(c)), MF, Bulk and CG approximations generate wrong density profiles, with strong oscillations instead of a convergence to the bulk. The positions of maxima from the wall are overestimated and they correspond to multiples of the weighting radius $\sigma$, for the CG approximation. The latter presents the worst prediction for the amplitude of the layers, and conduces to the underestimation of the adsorbed quantity, as 


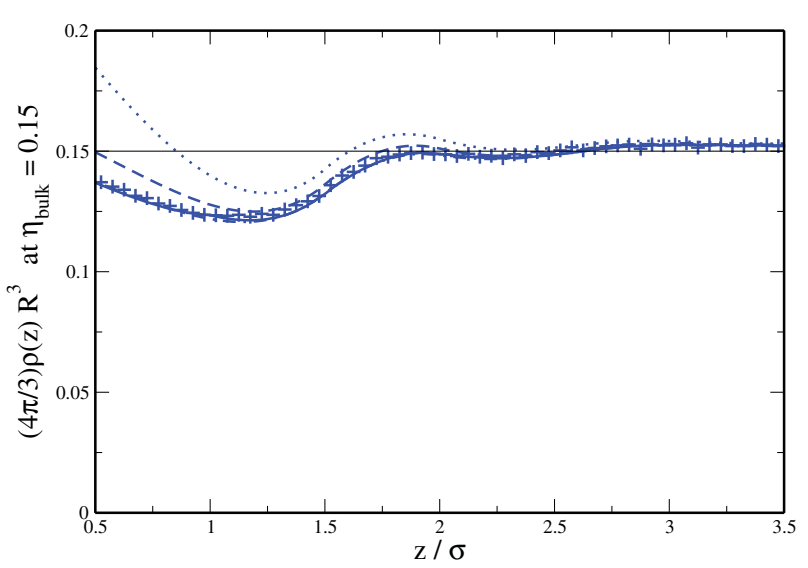

(a)

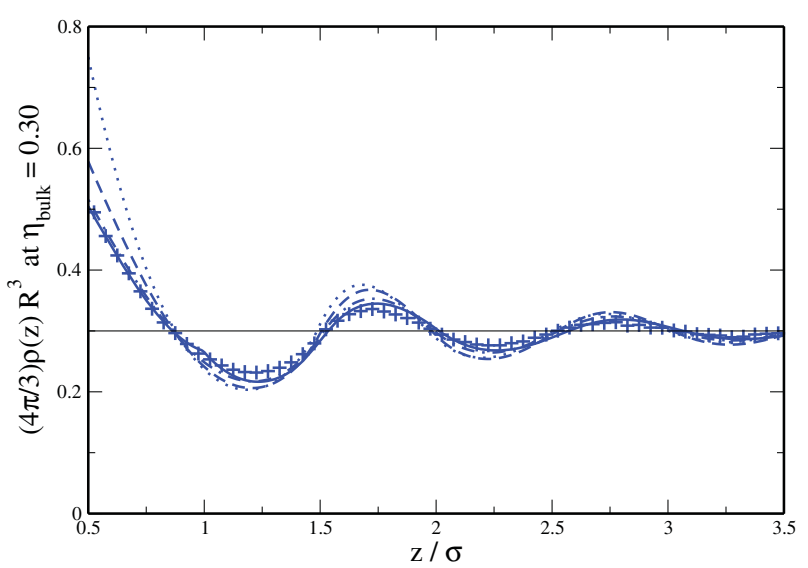

(b)

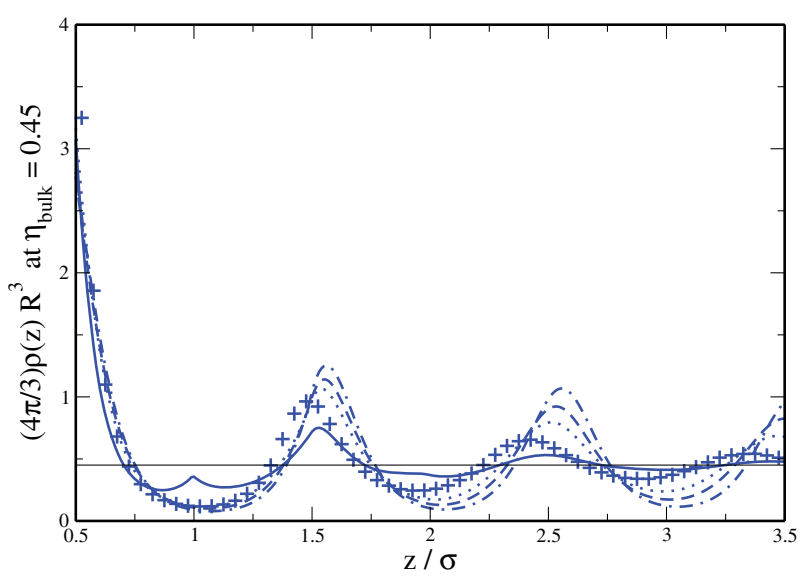

(c)

Figure 4. Density profiles of the pure square-well fluid studied. The variable $z$ represents the normal distance to the left hard planar wall placed at $z=0$. (a) $\eta_{\text {bulk }}=0.15$; (b) $\eta_{\text {bulk }}=0.30$; (c) $\eta_{\text {bulk }}=$ 0.45 .

seen above (see Figure 3). The result obtained with the KR-FMT approximation at high density is different from the others. It presents a non-physical peak at the radius of the weighting $R$ (and multiples), underestimates the

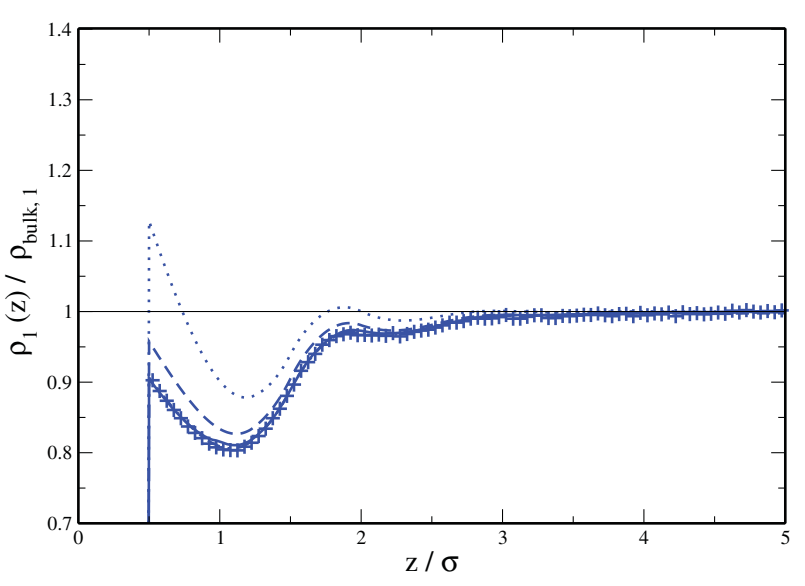

(a)

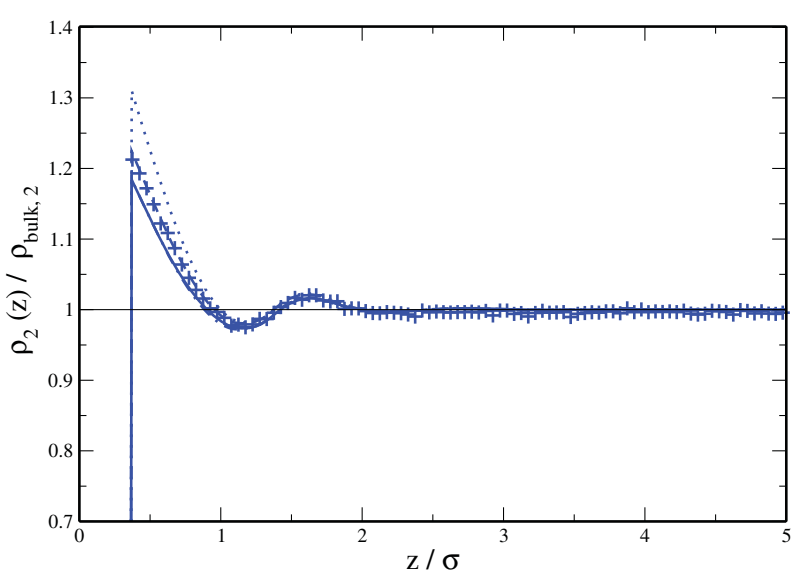

(b)

Figure 5. Density profiles of the binary square-well fluid studied. Density profiles for the square-well fluid studied. The variable $z$ represents the normal distance to the left hard planar wall placed at $z=0$. (a) Relative density profile of the molecule 1 ; (b) relative density profile of the molecule 2 .

amplitude of real peaks, but eventually converges to the bulk density.

Generally speaking, the position of the maxima depends on the weighting radius used in the different approximations. According to the system studied, some authors have introduced an adjustable parameter to fit the density profiles (see [5] for a recent example). Here, we do not follow this approach, and use only the square-well parameters for all the four approximations, in order to compare them. To conclude, the weighted term $\left(\bar{v}_{i k}^{(3)}(\vec{r})-\bar{n}_{i k}^{(3)}(\vec{r})\right)$ that characterises these DFT versions is responsible for the main structure of the fluid close to the wall. But, in order to improve the MF approximation estimations, one must add weighted densities into the pair correlation function. Important differences between these four DFT versions have been shown at very high 
density. In this case, the choice of the approximation leads to different qualitative and quantitative results.

\subsection{Density profiles of a square-well binary mixture}

If the square-well fluid model is not correct for pure fluids, it will be presumably worse for mixtures, because of the additional hypothesis on mixing rules present in all the terms of the free energy functional. This is why the approximations considered in this work are tested only at low density, for $\eta_{\text {total }}=0.15$, where the four models are qualitatively correct. The molecule of specie 1 in the mixture has the same parameters as in Section 4.1 , i.e. $k_{B} T / \varepsilon_{1}$ $=2$ and $\lambda_{1}=1.5$. We define the parameters of a molecule of specie 2, smaller and less attractive than the molecule 1 , with $R_{2} / R_{1}=0.75, \varepsilon_{2} / \varepsilon_{1}=2 / 3$ and $\lambda_{2}=1.3$. The molecular bulk fractions are $x_{1}=0.75$ and $x_{2}=0.25$. The other variables related to the system and the simulations are the same as that in the previous section.

The conclusions that can be drawn from the density profiles (Figure 5) for each approximation are the same than for pure fluids. The MF approximation presents important differences with the simulations near the wall, while the other approximations give better results. Because molecule 2 is less attractive than molecule 1, its profile resembles more of a hard-sphere profile, with a maximum at the wall contact distance. The selectivity

$$
\operatorname{Selectivity~}_{2 / 1}(z)=\left(\frac{\rho_{2}(z)}{\rho_{1}(z)}\right) /\left(\frac{\rho_{\text {bulk }, 2}}{\rho_{\text {bulk }, 1}}\right)
$$

provides another representation to test the quality of the mixture description. Figure 6 shows an important difference with simulations for the selectivity computed in the MF approximation, and some little differences

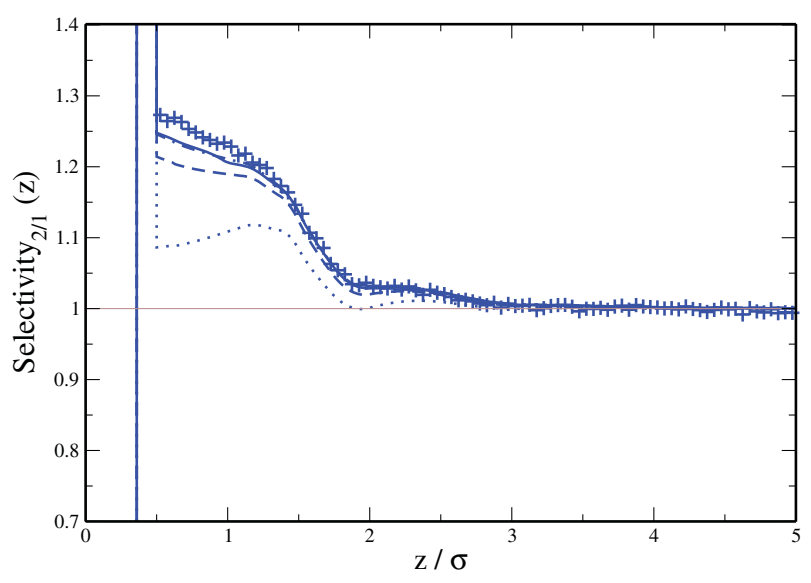

Figure 6 . Selectivity of the specie 2 w.r.t. the specie 1 . The variable $z$ represents the normal distance to the left hard planar wall placed at $z=0$. for the other approximations. The approximations with weighted densities (CG and KR-FMT) present similar results, with a very good global agreement with simulation data.

\section{Conclusion}

We have compared four inhomogeneous square-well fluid models, only distinct from the approximation on the pair correlation function $g_{i k \text {, eff }}^{\text {att }}(\vec{r})$, present in the attractive free energy term of the DFT. Various criteria can be considered to select or validate an approximation, depending on fundamental interests or for a specified application. In this case, the considered selection criteria are the fulfillment of the following conditions: verification of sum rules and accuracy in the prediction both adsorbed quantity and density profiles. Even if a model satisfies one of these criteria, it may fail to describe others, so they can be considered supplementary to each other and in no case redundant. Different conclusions can be drawn according to the bulk density value. In summary, the MF approximation satisfies sum rules but leads to the worst prediction of excess adsorption and density profiles. The Bulk approximation provides good qualitative results on density profiles but does not satisfy sum rules. The CG approximation presents good results for all the criteria except for the highest densities tested $(\eta>0.4)$, where the model fails. The KR-FMT approximation leads to the best results on sum rules for a model with a $g_{i k \text {,eff }}^{\text {att }}(\vec{r})$ different to unity, but reveals some non-desired peaks in the density profile at the highest densities. We recall that the quantitative results obtained here are specific to the square-well fluid studied, but the conclusions will not change with other fluid parameters and are qualitatively general.

According to this study, one can attach more importance to any of these criteria depending on the specific objectives intended for the model development and purpose. They can serve as references in the development of new DFT models, eventually with other criteria, according to the objective of the work and the accuracy desired. In particular, MF is a good first approximation for a lot of thermodynamic models [19]; and at low and medium densities, differences between CG and KR-FMT results for sum rules and density profiles are negligible, indicating that both of these approximations can be used to compare experimental adsorbed quantities and predictions from the theory [23].

\section{Acknowledgments}

M.M. Pineiro acknowledges CESGA (www.cesga.es) for providing access to computing facilities, and financial support by 
Ministerio de Economía y Competitividad, in Spain (Grant Ref. FIS2015-68910-P). T. Bernet acknowledges the Ministère de l'Éducation, de l'Enseignement Supérieur et de la Recherche, in France, for his MENRT Ph.D. Grant and Total SA for additional funding. We acknowledge R. Evans for his useful questions, remarks and discussions at the Thermodynamics 2017 conference. G. Jackson is gratefully acknowledged for the invitation to publish this work.

\section{Disclosure statement}

No potential conflict of interest was reported by the authors.

\section{Funding}

Ministerio de Economía y Competitividad, in Spain [grant number FIS2015-68910-P]; Ministère de l'Éducation, de l'Enseignement Supérieur et de la Recherche, in France; Total SA.

\section{References}

[1] R. Evans, in Fundamentals of Inhomogeneous Fluids, CRC Press, edited by D. Henderson, Marcel Dekker Inc, NewYork, (1992), pp. 85-175.

[2] Y. Rosenfeld, Phys. Rev. Lett. 63(9), 980-983 (1989).

[3] C. Malheiro, B. Mendiboure, F. Plantier, F.J. Blas, and C. Miqueu, J. Chem. Phys. 140(13), 134707 (2014).

[4] J. Gross, J. Chem. Phys. 131(20), 204705 (2009).

[5] E. Sauer and J. Gross, Ind. Eng. Chem. Res. 56(14), 41194135 (2017).

[6] R. Roth, J. Phys.: Condens. Matter 22(6), 063102 (2010).
[7] A. Gil-Villegas, A. Galindo, P.J. Whitehead, S.J. Mills, G. Jackson, and A.N. Burgess, J. Chem. Phys. 106(10), 4168-4186 (1997).

[8] E. Kierlik and M.L. Rosinberg, Phys. Rev. A 42(6), 33823387 (1990).

[9] T. Bernet, M.M. Piñeiro, F. Plantier, and C. Miqueu, J. Phys. Chem. C 121(11), 6184-6190 (2017).

[10] H. Hansen-Goos and R. Roth, J. Phys.: Condens. Matter 18(37), 8413 (2006).

[11] R. Evans, Adv. Phys. 28(2), 143-200 (1979).

[12] J.A. Barker and D. Henderson, J. Chem. Phys. 47(8), 2856-2861 (1967).

[13] A. Malijevský and S. Labík, Mol. Phys. 60(3), 663-669 (1987).

[14] A.J. Archer, B. Chacko, and R. Evans, J. Chem. Phys. 147(3), 034501 (2017).

[15] M. Schoen, A.J. Haslam, and G. Jackson, 33(42), 11345 (2017).

[16] G.J. Gloor, G. Jackson, F.J. Blas, E.M. del Río, and E. de Miguel, J. Chem. Phys. 121(24), 12740-12759 (2004).

[17] F. Llovell, A. Galindo, F.J. Blas, and G. Jackson, J. Chem. Phys. 133(2), 024704 (2010).

[18] J.K. Percus, J. Chem. Phys. 75(3), 1316-1319 (1981).

[19] H.T. Davis, Statistical Mechanics of Phases, Interfaces, and Thin Film, Wiley-VCH, New-York, (1996).

[20] N.F. Carnahan and K.E. Starling, J. Chem. Phys. 51(2), 635-636 (1969).

[21] S. Phan, E. Kierlik, M.L. Rosinberg, B. Bildstein, and G. Kahl, Phys. Rev. E 48(1), 618-620 (1993).

[22] Y.X. Yu and J. Wu, J. Chem. Phys. 116(16), 7094-7103 (2002).

[23] C. Malheiro, B. Mendiboure, J.M. Míguez, M.M. Piñeiro, and C. Miqueu, J. Phys. Chem. C 118(1), 24905-24914 (2014). 